\title{
ACURÁCIA DO POSICIONAMENTO ABSOLUTO GPS COM CORREÇÃO DA IONOSFERA ADVINDA DE MAPAS IONOSFÉRICOS GLOBAIS E REGIONAIS
}

\section{Accuracy of GPS Absolute Positioning with Ionosphere Correction from Global and Regional Ionosphere Maps}

\author{
Gilmara Dannielle de Carvalho Rocha ${ }^{1}$ \\ Haroldo Antonio Marques ${ }^{1}$ \\ João Francisco Monico Galera² \\ 1 Universidade Federal de Pernambuco-UFPE / Centro de Tecnologia e Geociências Departamento de \\ Engenharia Cartográfica Programa de Pós Graduação em Ciências Geodésicas e Tecnologias da \\ Geoinformação, Recife - PE - Brasil. rochagdc@gmail.com; haroldoh2o@gmail.com \\ 2Universidade Estadual Paulista-UNESP / Faculdade de Ciências e Tecnologia Pós Graduação em \\ Ciências Cartográficas Departamento de Cartografia, Presidente Prudente - SP. galera@fct.unesp.br
}

\section{Resumo:}

O efeito provocado pela ionosfera no sinal GPS é um dos mais impactantes no processo de estimativa das coordenadas geodésicas, principalmente para dados de simples frequência, sem o uso de um modelo ionosférico adequado. Neste caso, pode-se atualmente aplicar as correções ionosféricas advindas do modelo de Klobuchar, dos Mapas Globais (GIM) ou Mapas Regionais da Ionosfera. Sendo os dois últimos citados os que têm sido muito investigados pela comunidade cientifica. Nesse artigo destaca-se o LPIM (La Plata Ionospheric Model) desenvolvido na Universidade La Plata e disponibilizado dentro do contexto do projeto SIRGAS. Neste artigo apresenta-se a avaliação da acurácia do posicionamento absoluto com aplicação das correções ionosféricas advindas do GIM e do LPIM. Foram realizados processamentos no modo PPP utilizando somente código com dados GPS coletados em estações da RBMC. A estimativa das coordenadas foi realizada com solução época por época e comparadas com as coordenadas estimadas na rede SIRGAS-CON. Estimou-se o EMQ diário com geração de séries temporais para o ano de 2013. Com base nas melhorias produzidas com a utilização do modelo ionosférico regional, propõe-se a modificação do modelo estocástico do ajustamento, tendo em vista que somente o modelo funcional é afetado pelas correções ionosféricas advindas dos modelos.

Palavras-chave: Posicionamento absoluto GPS; Efeitos Ionosféricos; Mapas Globais e Regionais da Ionosfera

\section{Abstract:}

The effect caused by the ionosphere in the GPS signal is one of the most important in the geodetic coordinate estimation process, mainly for data collected by single frequency receivers, 
if a not consistent ionospheric model is available. For such case, one can currently apply the ionospheric corrections provided by the Klobuchar model and from Global or Regional Ionosphere Maps. The latter have been investigated by the scientific community in recent years. In this this paper, attention is driven to the LPIM (La Plata Ionospheric Model) developed at the University La Plata and made available within the SIRGAS project context. Thus, this paper presents the evaluation of the absolute positioning accuracy with ionospheric corrections coming from the GIM and LPIM. It was accomplished PPP processing by using only GPS code data collected by Brazilian RBMC stations. The coordinates estimation was carried out in an epoch by epoch basis and the solutions were compared with ground truth estimated in the SIRGASCON network providing the daily RMS with time series generation for the complete year of 2013. Based on the improvement produced by the application of the LPIM it is proposed the stochastic model modification for the adjustment considering that only the functional model is affected by ionospheric corrections coming from distinct models.

Keywords: Absolute GPS Positioning; Ionosphere Effects; Global and Regional Ionosphere Maps.

\section{Introdução}

As observáveis GNSS (Global Navigation Satellite System) estão sujeitas a diversos efeitos sistemáticos dentre os quais se destaca aquele relacionado com o atraso provocado pela ionosfera. A camada da ionosfera é caracterizada como um meio dispersivo composta por íons e elétrons livres, que afetam a propagação de ondas eletromagnéticas, as quais são transmitidas pelos satélites (Aguiar, 2005; Matsuoka, 2007; Monico, 2008). Outros efeitos incluem o atraso provocado pela troposfera, carga da atmosfera, multicaminho, perdas de ciclos, fase windup, efeitos relativísticos e aqueles relacionados com a estação, como por exemplo, erro proveniente do centro de fase das antenas das estações, movimento das placas tectônicas, marés terrestres e oceânicas (Seeber, 2003; Leick, 2004; Monico, 2008; Marques, 2012).

No caso de posicionamento geodésico no modo relativo considerando linhas de base curtas, a maioria dos efeitos da ionosfera e outros são teoricamente eliminados ao aplicar a combinação de Dupla Diferença (DDs) no ajustamento dos dados. Porém, o mesmo não ocorre para longas linhas de base, com comprimento acima de $100 \mathrm{~km}$, onde os efeitos da ionosfera tornam-se menos correlacionados nas estações envolvidas. Em se tratando de posicionamento absoluto com receptores de simples frequência, seja Posicionamento por Ponto Simples (PP) ou Posicionamento por Ponto Preciso (PPP), uma estratégia adequada de correção dos efeitos ionosféricos deve ser adotada.

Quando se têm dados GNSS de dupla frequência pode-se aplicar a combinação linear livre da ionosfera (ion-free). Esta combinação permite eliminar os efeitos de primeira ordem da ionosfera, os quais representam em torno de $99 \%$ do atraso total provocado nas medidas, sendo que o restante fica a cargo dos efeitos de ordem superior (Marques et al., 2011; Marques et al., 2014). Para usuários que dispõem de receptores de simples frequência, outras estratégias devem ser adotadas, como por exemplo, o uso do modelo de Klobuchar que proporciona correção de aproximadamente $50 \%$ do efeito total da ionosfera ou a aplicação da correção ionosférica a partir dos Mapas Globais (GIM - Global Ionosphere Maps) ou Regionais da Ionosfera (RIM Regional Ionosphere Maps) ou ainda a estimativa dos efeitos residuais da ionosfera (Klobuchar, 1996; Schaer et al., 1998; Brunini, 1998; Matsuoka, 2003). 
Os Mapas Globais da Ionosfera podem ser obtidos nos centro que contribuem com o IGS (International GNSS Service), como por exemplo, o CODE (Center for Orbit Determination), ESA (European Space Agency), JPL (Jet Propulsion Laboratory) e UPC (Polytechnic University of Catalonia). Esses mapas são fornecidos no formato IONEX (IONosphere map Exchange format) com valores de VTEC (Conteúdo Total de Elétrons na direção vertical) distribuídos em grades (grids) com resolução espacial $5^{\circ}$ x 2,5 em longitude e latitude, respectivamente, e 2 horas de resolução temporal (Schaer et al.,1998; IGS, 2014). Em relação aos mapas regionais, estes têm sido alvo de investigações da comunidade científica nacional e internacional nos últimos anos, podendo-se citar, por exemplo, os desenvolvimentos no INPE (Instituto Nacional de Pesquisas Espaciais) com o projeto EMBRACE (Estudo e Monitoramento Brasileiro do Clima Espacial), o desenvolvimento do modelo Mod_Ion da UNESP (Universidade Estadual Paulista) (Camargo, 1999) e o LPIM o qual vem sendo desenvolvido na UNLP (Universidad Nacional de La Plata), sendo este último disponibilizado dentro do contexto do projeto SIRGAS (Sistema de Referência Geocêntrico para as Américas) (Brunini et al., 2008).

Para a estimativa de VTEC no caso dos mapas regionais, geralmente, utilizam-se dados GNSS coletados em uma rede geodésica mais densa (maior número de estações) na região. Além disto, os mapas regionais são disponibilizados com resolução temporal e espacial maior que os mapas globais de forma que se esperam valores mais realísticos de VTEC para a região proporcionando melhorias em termos de posicionamento. No caso do LPIM, utilizam-se dados GNSS coletados na rede SIRGAS e os valores de VTEC são disponibilizados em arquivos horários com resolução espacial de $1^{\circ} \times 1^{\circ}$ de latitude e longitude, respectivamente.

É importante destacar que o Brasil por estar localizado na região equatorial sofre forte influência dos efeitos ionosféricos, o que requer modelos mais apropriados abrindo um campo de estudos e investigações relacionado com o tema clima espacial. Essa região do planeta sofre influência da anomalia equatorial e é considerada uma das que apresentam maiores variações temporais e espaciais do TEC, além da ocorrência dos efeitos de cintilação ionosférica (Matsuoka, 2007; Silva, 2009).

Dentro deste contexto, este trabalho apresenta a avaliação do modelo LPIM e GIM com as correções da ionosfera derivadas destes modelos e aplicadas no posicionamento GPS absoluto. Foram realizados processamentos utilizando dados do código C/A coletados em estações da RBMC (Rede Brasileira de Monitoramento Contínuo). Os dados foram processados no software RT_PPP (Real time PPP) desenvolvido por Marques (2012) e as coordenadas estimadas foram comparadas com aquelas advindas da solução semanal SIRGAS-CON, as quais são dadas atualmente em ITRF2008 (International Terrestrial Reference Frame - 2008).

A estimativa das coordenadas foi realizada com solução época por época considerando o uso de órbitas precisas, correções de relógios dos satélites e diversos outros efeitos que devem ser corrigidos para a realização de PPP. As análises foram realizadas em termos de EMQ (Erro Médio Quadrático) diário com geração de séries temporais para o ano de 2013. Com base nas melhorias produzidas com a utilização do modelo ionosférico regional foi proposta a modificação do modelo estocástico do ajustamento tendo em vista que somente o modelo funcional é afetado quando se aplica as correções ionosféricas derivadas de diferentes modelos ionosféricos. A revisão bibliográfica relacionada ao assunto, bem como os resultados e análises envolvendo o uso de modelos regionais e globais no posicionamento absoluto são apresentados neste artigo. 


\section{Modelo funcional e estocástico do posicionamento absoluto}

O posicionamento absoluto GNSS é denominado PP ou PPP, sendo que o primeiro se refere à solução de navegação obtidos das observações das medidas de código e o segundo requer o uso de dados de código e fase com correção de praticamente todos os efeitos envolvidos com a propagação dos sinais na atmosfera e efeitos geodinâmicos relacionados com a estação. $\mathrm{O}$ ajustamento das observações coletadas pelos receptores é realizado, em geral, na forma recursiva, haja vista o número de observações coletadas num determinado espaço de tempo (Seeber, 2003; Leick, 2004; Hoffman Wellenhof et al, 2001; Monico, 2008; Marques, 2012).

O modelo matemático funcional e estocástico para o ajustamento de medidas GPS é amplamente divulgado na literatura podendo se citar Seeber (2003); Leick (2004); Monico (2008); Hoffman Wellenhof et al. (2001). Para esse trabalho, aplicou-se o ajustamento com solução época por época (cada época solucionada independentemente) para realização do método PPP, porém utilizando somente medidas do código C/A. A Equação 1 de pseudodistância, em metros, derivada do código C/A $\left(\mathrm{PD}_{\mathrm{rL1}}^{\mathrm{s}}\right)$ é apresentada a seguir:

$$
\mathrm{PD}_{\mathrm{r} L 1}^{s_{\mathrm{i}}}=\rho_{\mathrm{r}}^{s_{\mathrm{i}}}+\mathrm{c}\left(\mathrm{dt}_{\mathrm{r}}-\mathrm{dt}{ }^{s_{\mathrm{i}}}\right)+\mathrm{I}_{\mathrm{r} L 1}^{s_{\mathrm{i}}}+\mathrm{T}_{\mathrm{r}}^{s_{\mathrm{i}}}+\mathrm{dm}_{\mathrm{r}}^{s_{\mathrm{i}}}+O \mathrm{Orb}^{s_{\mathrm{i}}}+\mathrm{b}_{\mathrm{r}}+\mathrm{b}^{s_{\mathrm{i}}}+v_{\mathrm{PD}_{\mathrm{i}}^{s_{\mathrm{i}}}}
$$

onde: ${ }^{\rho_{\mathrm{s}}^{s}}$ é a distância geométrica entre o satélite $\left(\mathrm{s}_{\mathrm{i}}\right)$ no instante de transmissão e o receptor (r) no instante de recepção do sinal; c é a velocidade da luz no vácuo; ${ }^{\mathrm{dt}_{\mathrm{r}}} \mathrm{e}^{\mathrm{dt}^{\mathrm{s}}}$ representam os erros dos relógios do receptor e satélite, respectivamente; ${ }^{\mathrm{I}_{\mathrm{rL} 1}^{\mathrm{s}}}$ é o atraso provocado pela ionosfera na frequência L1; $\mathrm{T}_{\mathrm{r}}^{s_{i}}$ é o atraso provocado pela troposfera; ${ }^{d m^{5}}$ representa o efeito de multicaminho; $\mathrm{Orb}^{\mathrm{s}_{\mathrm{i}}}$ é o erro da órbita do satélite; ${ }^{\mathrm{b}_{\mathrm{r}}} \mathrm{e}^{\mathrm{b}^{\mathrm{s}}}$ são os atrasos de hardware para o receptor e satélite,

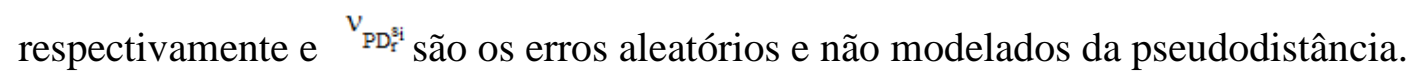

$\mathrm{O}$ atraso de hardware, seja no satélite ou no receptor, é causado pela diferença entre os caminhos percorridos pelas portadoras L1 e L2 no hardware. Os atrasos (bias instrumentais) Bc1, Bp1 e $\mathrm{Bp} 2$ são relacionados, respectivamente aos códigos $\mathrm{C} 1$, P1 e P2. Com a modernização do GPS, os novos satélites transmitem o novo código L2C na L2 onde comparece o atraso BC2 e o mesmo ocorre com o código L5C presente no novo sinal L5 transmitido pelo GPS. Estes atrasos não são acessíveis diretamente em sua forma absoluta, ou seja, eles são obtidos quando se faz combinações entre as portadoras (forma relativa). Desta maneira, os atrasos ou bias são denominados de DCB (Differential Code Bias) e, para o caso envolvendo dados nas portadoras

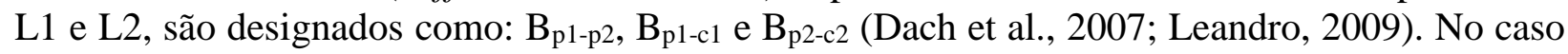
do hardware dos satélites, a magnitude desse atraso é determinada durante o processo de calibração dos satélites antes do seu lançamento e é conhecida como TGD (Timing Group Delay), cujos valores para cada satélite fazem parte das mensagens de navegação (Wilson et al., 1999). O valor de $B_{\mathrm{p} 1-\mathrm{p} 2}$ pode ser relacionado com o atraso de grupo TGD da seguinte forma a qual é apresentada na Equação 2:

$$
\mathrm{TGD}=-1,5457 \mathrm{~B}_{\mathrm{pl}-\mathrm{p} 2}+\mathrm{B}_{0}
$$

onde, $\mathrm{B}_{0}$ é um valor arbitrário irrelevante para muitas aplicações (Dach et al., 2007).

O efeito da troposfera pode ser corrigido a partir de modelos matemáticos, como por exemplo, modelos de Hopfield e de Saastamoinen ou a partir de modelos advindos de dados de PNT 
(Previsão Numérica do Tempo) (Sapucci, 2006). A órbita do satélite pode ser calculada a partir das efemérides transmitidas (mensagens de navegação) ou das precisas disponibilizadas, por exemplo, no site do IGS. O efeito da ionosfera, objeto de avaliação deste trabalho, pode ser corrigido a partir de modelos matemáticos ou fazendo uso de mapas globais ou regionais da ionosfera. Dessa forma, os parâmetros incógnitos envolvidos na equação 1 são as coordenadas da estação e o erro do relógio do receptor. A distância geométrica é calculada a partir da diferença das coordenadas do satélite (no instante de transmissão $-t^{t}$ ) e do receptor $\left(X_{r}, Y_{r}\right.$ e $Z_{r}$ ), apresentada na Equação 3:

$$
\rho_{\mathrm{r}}^{s}=\sqrt{\left(\mathrm{X}^{s}\left(\mathrm{t}^{\mathrm{t}}\right)-\mathrm{X}_{\mathrm{r}}\right)^{2}+\left(\mathrm{Y}^{s}\left(\mathrm{t}^{\mathrm{t}}\right)-\mathrm{Y}_{\mathrm{r}}\right)^{2}+\left(\mathrm{Z}^{\mathrm{s}}\left(\mathrm{t}^{\mathrm{t}}\right)-\mathrm{Z}_{\mathrm{r}}\right)^{2}}
$$

A linearização por série de Taylor da equação de pseudodistância (Equação 1), considerando o receptor $(\mathrm{r})$ e o satélite $\left(\mathrm{s}_{\mathrm{i}}\right)$ pode ser escrita da seguinte forma (Equação 4) (Monico, 2008):

$$
E\left\{\Delta P D_{\mathrm{r} L 1}^{s_{\mathrm{i}}}\right\}=-\frac{\mathrm{X}^{s_{\mathrm{i}}}-\mathrm{X}_{\mathrm{r}}^{0}}{\left(\rho_{\mathrm{r}}^{s_{\mathrm{i}}}\right)^{\rho}} \Delta \mathrm{X}_{\mathrm{r}}-\frac{\mathrm{Y}^{\mathrm{s}_{\mathrm{i}}}-\mathrm{Y}_{\mathrm{r}}^{0}}{\left(\rho_{\mathrm{r}}^{s_{\mathrm{i}}}\right)^{0}} \Delta \mathrm{Y}_{\mathrm{r}}-\frac{\mathrm{Z}^{\mathrm{s}_{\mathrm{i}}}-\mathrm{Z}_{\mathrm{r}}^{0}}{\left(\rho_{\mathrm{r}}^{s_{\mathrm{i}}}\right)^{0}} \Delta \mathrm{Z}_{\mathrm{r}}+\mathrm{c}\left(\mathrm{cdt}_{\mathrm{r}}\right)
$$

$\mathrm{Na}$ Equação 4, E\{.\} representa o operador de esperança matemática; ${ }^{\Delta \mathrm{PD}_{\mathrm{I}}^{\mathrm{s}} \mathrm{s}}$ é a diferença entre a pseudodistância observada e a calculada (vetor L para pseudodistância); ( $\left.\rho_{\text {ș }}^{\tilde{s}}\right)^{0}$ é distância geométrica calculada em função dos parâmetros aproximados. Os termos ( $\left.\Delta \mathrm{X}_{\mathrm{r}}, \Delta \mathrm{Y}_{\mathrm{r}}, \Delta \mathrm{Z}_{\mathrm{r}} \mathrm{e} \mathrm{dt}_{\mathrm{r}}\right)$ são as correções aos parâmetros incógnitos, ou seja, as coordenadas da estação e erro do relógio do receptor. A Equação 4 linearizada na forma matricial $(E\{L\}=A X)$ pode ser escrita como é expressa na Equação 5:

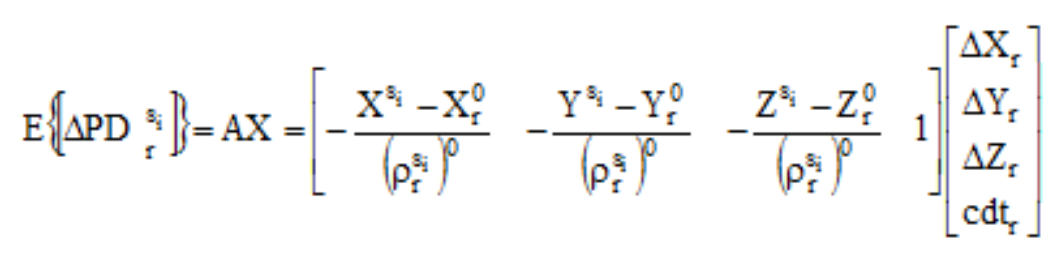

O modelo estocástico para o caso do posicionamento absoluto é composto pela MVC (Matriz de Variância e Covariância) de Lb, geralmente, na forma diagonal, apresentada na Equação 6:

$$
\Sigma_{L b}=\left[\begin{array}{ccccc}
\sigma^{2} \Gamma_{L i}^{s 1} & & & & 0 \\
& \sigma^{2} L_{L i}^{s 2} & & & \\
& & \sigma^{2} L_{L i}^{53} & & \\
& & & \ddots & \\
0 & & & & \sigma^{2}{ }_{\mathrm{PL}}^{s a}
\end{array}\right]
$$

Estratégias de ponderação em função do ângulo de elevação do satélite (elev) podem ser adotadas tais como descritas por Silva (2009). Para esse trabalho considerou-se o valor da precisão da medida adotado pelo usuário, a qual é ponderada pela função inverso do seno do ângulo de elevação do satélite. Outra estratégia adotada consiste em considerar de forma adicional a precisão da ionosfera calculada a partir do VTEC interpolado, seja do mapa regional ou global. Dessa forma a precisão da pseudodistância ( $\left.{ }^{{ }{ }^{\mathrm{SD}} \mathrm{Si}}\right)$ é representada pela Equação 7: 


$$
\sigma_{\mathrm{PD}}^{2} D_{\mathrm{Li}}^{\mathrm{Si}}=\frac{1}{\operatorname{sen}(\mathrm{elev})} \sigma_{\mathrm{C} / \mathrm{A}}^{2}+\sigma_{\mathrm{IONO}}^{2}
$$

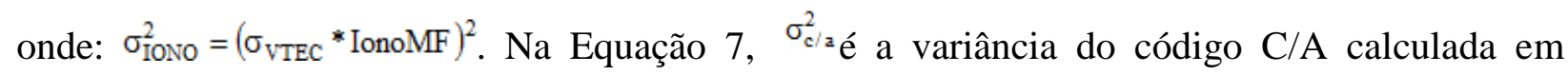

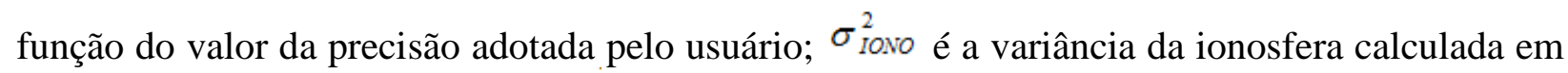
função da incerteza do VTEC ( ${ }^{{ }_{\text {VTEC }}}$ ) obtido a partir do mapa ionosférico, o qual é mapeado para a direção receptor satélite através da função de mapeamento IonoMF (ver seção 3). O valor de $\sigma_{\mathrm{VTEC}}$ pode ser obtido com base em valores divulgados pelo órgão gerador do mapa ou a partir de valores disponibilizados no próprio mapa, os quais nem sempre estão disponíveis.

Experimentos foram realizados neste trabalho visando avaliar os valores a serem adotados para a utilização de mapas regionais e mapas globais da ionosfera.

\section{Introdução ao GIM e LPIM}

Os Mapas Globais da Ionosfera do IGS são produzidos a partir da combinação de dados de quatro centros de análises (CODE, ESA, JPL e UPC) e são disponibilizado em formato IONEX com valores de VTEC distribuídos em uma grade bidimensional de resolução espacial $5^{\circ} \times 2,5^{\circ}$ em longitude e latitude, respectivamente. A resolução temporal de cada mapa é de 2 horas e as grades são distribuídas de $87,5^{\circ}$ (Norte) até $87,5^{\circ}$ (Sul) em latitude e de $180^{\circ}$ (Oeste) a $180^{\circ}$ (Leste) em longitude. Além dos valores de VTEC, o GIM fornece os atrasos de hardware (DCB) para o código P1-P2 e P1-C1. Estes mapas podem ser obtidos no endereço eletrônico: ftp://cddisa.gsfc.nasa.gov/gps/products/ionex/ (Schaer, 1999; Liu, 2001; IGS, 2014).

A precisão do VTEC disponível no GIM varia em função da região do globo, do número de estações GNSS utilizadas, do período em que os dados (alta ou baixa atividade ionosférica) se encontram, dentre outros fatores. O intervalo divulgado de variação da precisão do VTEC é de 2 a 8 TECU (CODE, 2014; Van Bree et al., 2012). No caso da região brasileira, dados de oito estações da rede IGS fazem parte da estimativa do VTEC, sendo elas: BRAZ (Brasília), BRFT (Eusébio), CHPI (Cachoeira Paulista), POVE (Porto Velho), RECF (Recife), SALU (São Luís), SAVO (Salvador) e UFPR (Curitiba).

Desde 2005, o centro de processamento de La Plata (CPLAT) utiliza o Modelo Ionosférico de La Plata (LPIM) aplicado a 50 estações GPS localizado na América do Sul, para gerar diariamente mapas ionosféricos. O VTEC é estimado considerando o sistema de referência solargeomagnético com aplicação de harmônicos esféricos. O mapeamento do VTEC para a direção receptor satélite é realizado considerando o caso de uma camada infinitesimal da ionosfera (SIRGAS, 2014; CPLAT, 2014). Para o mapeamento do VTEC utiliza-se a hora local e latitude modificada, a qual permite suavizar a variabilidade espacial do VTEC, especialmente em regiões de baixa latitude com é o caso da região sul-americana.

Os mapas do LPIM são produzidos com resolução espacial de $1^{\circ}$ em latitude e longitude e sua região de cobertura varia de $-61^{\circ}$ até $30^{\circ}$ em latitude e de $-110^{\circ}$ a $-20^{\circ}$ em longitude com resolução temporal de 1 hora (Brunini et al, 2008). A Figura 1 apresenta a região geográfica de cobertura do LPIM, onde os pontos amarelos representam a localização das estações GNSS utilizada na estimativa dos valores de VTEC no LPIM. 


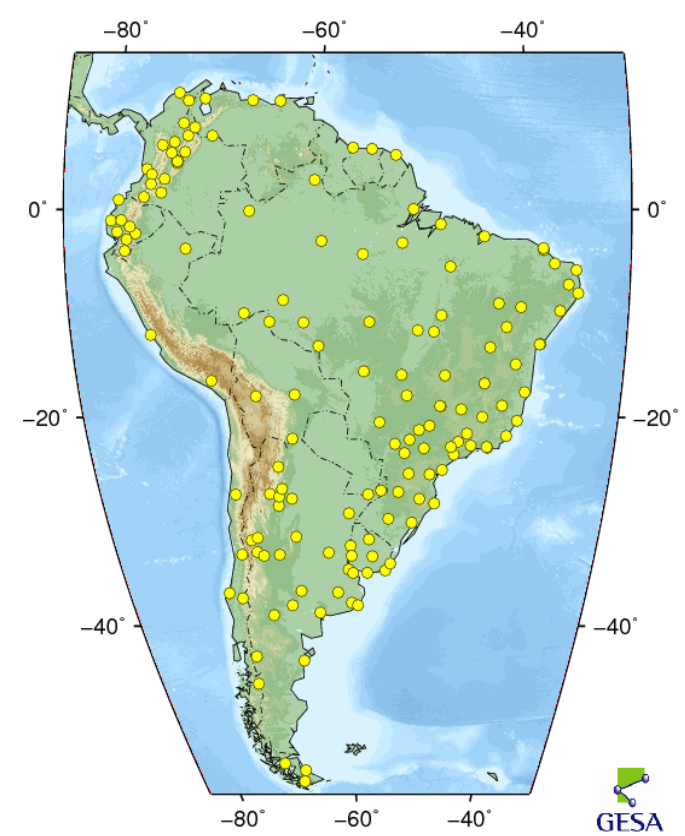

Figura 1: Região geográfica de cobertura do LPIM. Fonte: http://cplat.fcaglp.unlp.edu.ar.

Enquanto o GIM é disponibilizado em formato IONEX, os valores de VTEC do LPIM são disponibilizados em grades numéricas organizadas em arquivos horários para cada dia do ano.

Nos mapas da ionosfera tanto global quanto regional, os valores de VTEC são distribuídos em grade variando em função da latitude e da longitude, sendo necessário realizar um procedimento de interpolação considerando a variação temporal e espacial com o objetivo de obter o valor de VTEC mais próximo do da estação a qual se pretende determinar as coordenadas geodésicas. No caso desse trabalho os valores de VTEC do GIM e LPIM foram interpolados utilizando interpolação bilinear no espaço e linear para o tempo. Para cada grade com resolução espacial de $5^{\circ} \times 2,5^{\circ}$ no caso do GIM e $1^{\circ} \times 1^{\circ}$ no LPIM, armazenou-se quatro valores de VTEC próximo à localização geográfica de interesse como exemplificado na Figura 2. A interpolação para cada mapa é realizada considerando a latitude e longitude do ponto ionosférico de cada satélite na altura da camada infinitesimal da ionosfera. A Figura 2 apresenta interpolação do VTEC, onde o círculo azul são as quatro posições da grade e o círculo vermelho a posição de interesse. O IPP é o ponto ionosférico localização na camada da ionosfera onde o sinal GPS intercepta a camada.

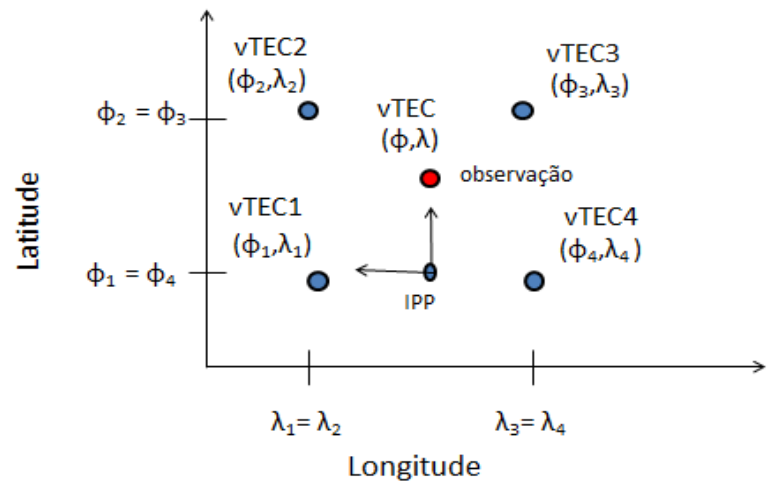

Figura 2: Interpolação do VTEC para localização geográfica do usuário.

Para converter valores de VTEC medidos no ponto ionosférico em STEC (slant TEC - TEC inclinado) na direção entre o satélite e o receptor utiliza-se a seguinte função de mapeamento, apresenta na Equação (8) (Camargo, 1999; Hofmann-Wellenhof et al., 2001): 
Nesta expressão, IonoMF $=1 / \cos \left(z^{\prime}\right)$ que representa a função de mapeamento, sendo $z$ ' é o ângulo zenital do caminho do sinal sobre o ponto ionosférico, o qual é calculado a partir da Equação 9:

$$
z^{\prime}=\operatorname{arcsen}\left(\frac{r_{m}}{r_{m}+h_{m}} \operatorname{sen}(z)\right)
$$

Tem-se que $\mathrm{r}_{\mathrm{m}}$ é o raio médio da Terra, $\mathrm{h}_{\mathrm{m}}$ representa a altura média do pico do perfil vertical da densidade de elétrons da ionosfera, que geralmente varia entre 300 a $450 \mathrm{~km}$ e $z$ é o ângulo zenital do satélite $\left(\mathrm{z}=90^{\circ}\right.$ - el, onde "el" é o ângulo de elevação do satélite). O cálculo do efeito ionosférico na portadora L1 é realizado com base no STEC e pode ser escrito da seguinte forma (Equação 10):

$$
\mathrm{I}_{\mathbf{r}}^{\mathrm{s}} \cong \frac{40,3}{\mathrm{f}_{1}^{2}} \mathrm{STEC}
$$

onde, $\mathrm{f}_{1}$ representa a frequência na portadora L1. A correção da ionosfera obtida conforme apresentada nesta seção foi aplicada no processamento dos dados GPS no modo posicionamento por ponto e os resultados e análises serão apresentados na próxima seção.

\section{Resultados}

Para avaliar a acurácia do posicionamento por ponto ao aplicar correções da ionosfera proveniente dos mapas ionosféricos globais e regionais, duas estratégias de peso foram adotadas nos processamentos dos dados, ou seja, aplicou-se o processamento com mesma estratégia de peso e com diferente peso para o caso do uso dos diferentes mapas.

Os dados GPS foram processados no RT_PPPP e os mapas horários do LPIM foram convertidos em formato IONEX utilizando um aplicativo 'lpim2IONEX' desenvolvido em cooperação entre o IBGE (Instituto Brasileiro de Geografia e Estatística) e o Departamento de Engenharia Cartográfica da UFPE (Universidade Federal de Pernambuco). Os efeitos da troposfera foram corrigidos a partir do modelo de Hopfield com valores de pressão e temperatura obtidos a partir da função GPT (Global Pressure and Temperature) (BOEHM et al., 2006), além disto, foram aplicados correção de diversos efeitos tais como Marés Oceânicas e Terrestres, PCV (Phase Center Variation - Centro de Fase das Antenas) e DCB (Differential Code Bias). As órbitas foram interpoladas das efemérides precisas do IGS e aplicaram-se correções precisas dos erros dos relógios dos satélites. Foram utilizados dados GPS de dez estações da RBMC no Brasil cuja distribuição geográfica pode ser vista na Figura 3. 


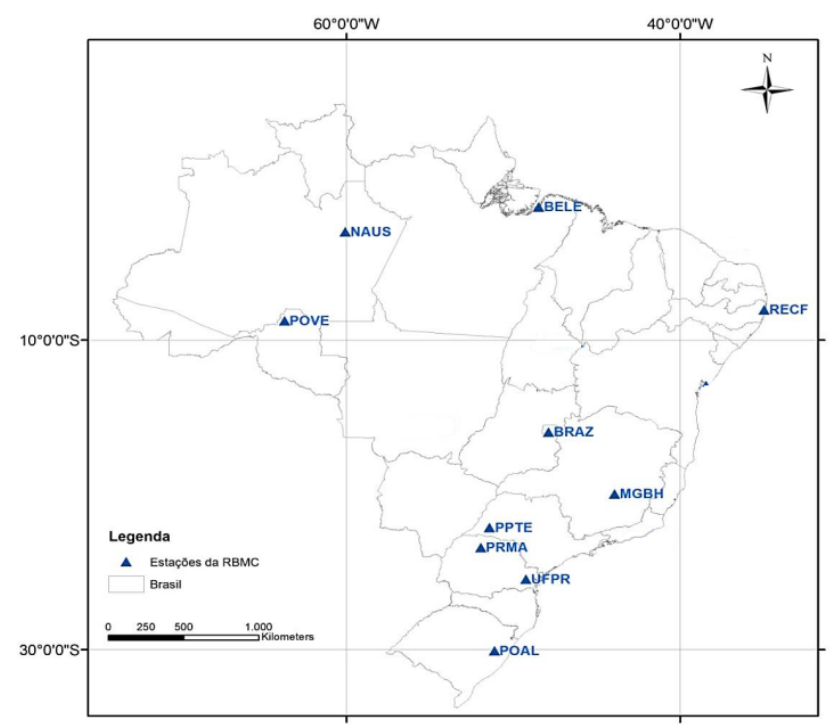

Figura 3: Mapa de localização das estações utilizadas nos experimentos.

O ajustamento dos dados GPS foi realizado com solução época por época (solução em cada época independente uma da outra) e obteve-se para cada dia uma média das coordenadas estimadas fornecendo assim uma solução diária. As coordenadas diárias estimadas na época de referência das órbitas precisas foram comparadas com a solução semanal da rede SIRGAS-CON e as discrepâncias obtidas foram convertidas para o Sistema Geodésico Local (SGL), o qual é denominado doravante de 'erros' no SGL. As análises são realizadas em termos de Erro Médio Quadrático (EMQ) diário ou anual. As análises envolvendo a mesma estratégia de ponderação para a aplicação do modelo GIM e LPIM no posicionamento são apresentadas na próxima seção.

\subsection{Análise do posicionamento aplicando GIM e LPIM}

No caso deste experimento, os dados GPS foram processados utilizando somente a precisão adotada pelo usuário para a montagem da matriz dos pesos, seja para a aplicação da correção ionosférica advinda do GIM ou do LPIM. Contudo, o valor da precisão é ponderado pelo inverso do seno do ângulo de elevação do satélite. A precisão do código C/A neste caso foi adotada com valor de 0,80 m. A Figura 6 mostra os erros para as componentes Este (DE), Norte (DN) e Altura (DU) no SGL (Sistema Geodésico Local) para cada época processada com dados coletados na estação NAUS para os dias 76 (17/03/2013) e 78 (19/03/2013) de 2013, cujo índice KP atingiu valores máximos, respectivamente de 6 (ionosfera ativa) e 2 (ionosfera calma) como mostra a Figura 4. Esse período foi escolhido de forma a apresentar o comportamento da ionosfera em duas situações extremas com a ionosfera ativa e calma.

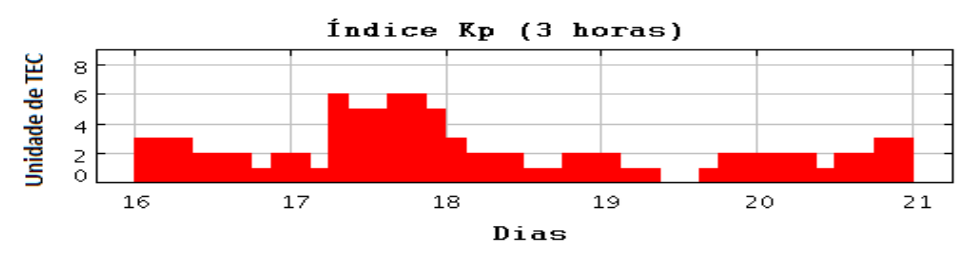

Figura 4: Índice Kp para os dias de março. Fonte: Adaptado de http://spidr.ngdc.noaa.gov/spidr. 


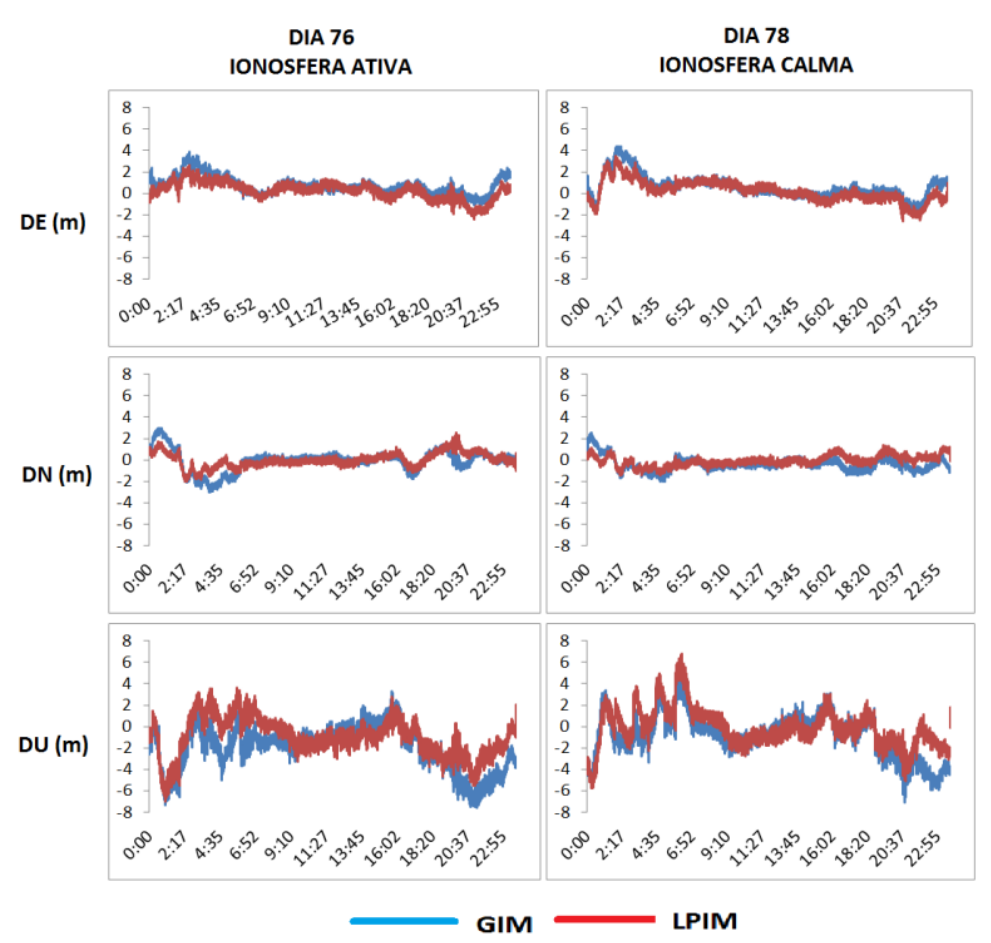

Figura 5: Série temporal do erro diário no SGL em DE, DN e DU para a estação NAUS.

A partir da Figura 5 verifica-se que, em geral, a aplicação do LPIM forneceu menores erros no SGL para cada época processada nos dias 76 e 78 de 2013 sendo que a componente altimétrica (DU) da estação NAUS é a mais afetada. O EMQ diário para cada componente é apresentado na Tabela 1, juntamente com a melhoria em termos percentuais ao utilizar o LPIM ao invés do GIM para correção da ionosfera no posicionamento.

Tabela 1: EMQ diário para os dias 76 e 78 na estação NAUS

\begin{tabular}{c|c|c|c|c|c|c}
\hline DIAS & MAPAS & DE $(\mathrm{m})$ & DN $(\mathrm{m})$ & DU $(\mathrm{m})$ & 2D $(\mathrm{m})$ & 3D $(\mathrm{m})$ \\
\hline \multirow{3}{*}{$\begin{array}{c}\text { DIA 76 } \\
\text { IONOSFERA ATIVA }\end{array}$} & GIM & 1,078 & 1,011 & 2,897 & 1,478 & 3,253 \\
\cline { 2 - 7 } & LPIM & 0,829 & 0,667 & 2,055 & 1,064 & 2,314 \\
\cline { 2 - 7 } & MELHORIA (\%) & 23,097 & 34,037 & 29,069 & 28,014 & 28,850 \\
\hline \multirow{2}{*}{$\begin{array}{c}\text { DIA 78 } \\
\text { IONOSFERA CALMA }\end{array}$} & GIM & 1,206 & 0,740 & 2,186 & 1,415 & 2,604 \\
\cline { 2 - 7 } & LPIM & 1,002 & 0,493 & 1,822 & 1,117 & 2,137 \\
\cline { 2 - 7 } & MELHORIA (\%) & 16,945 & 33,274 & 16,671 & 21,073 & 17,946 \\
\hline
\end{tabular}

Verifica-se na Tabela 1 que ao aplicar à correção ionosférica advinda LPIM as melhorias diárias variaram de 23\% (componente DE), 34\% (componente DN) e 29\% (componente DU) com ionosfera ativa e de $17 \%$ (DE), 33\% (DN) e 17\% (DU) com ionosfera calma. Para o caso da resultante $3 \mathrm{D}$, os melhoramentos foram de aproximadamente $29 \%$ e $18 \%$, respectivamente para os dias 76 e 78 .

O EMQ 3D diário e o melhoramento (média e máxima) do LPIM em relação o GIM para o processamento de cada uma das dez estações RBMC considerando dados do ano de 2013 é apresentado na Tabela 2 e Figura 6. A Tabela 2 apresenta a média do EMQ 3D considerando todos os dias do ano 2013 (EMQ 3D anual), bem como a melhoria média e máxima ao longo do ano. 
Tabela 2: EMQ 3D anual e melhoramento no posicionamento com aplicação do GIM e LPIM

\begin{tabular}{|c|c|c|c|c|}
\hline \multirow[t]{2}{*}{ ESTAÇÃO } & \multicolumn{2}{|c|}{ EMQ 3D MÉDIO (m) } & \multirow{2}{*}{$\begin{array}{l}\text { MÉLHORIA } \\
\text { MÉDIA (\%) }\end{array}$} & \multirow{2}{*}{$\begin{array}{l}\text { MELHORIA } \\
\text { MÁXIMA (\%) }\end{array}$} \\
\hline & GIM & LPIM & & \\
\hline BELE & 1,839 & 1,605 & 12,728 & 37,007 \\
\hline BRAZ & 1,840 & 1,761 & 4,264 & 22,707 \\
\hline MGBH & 1.907 & 1,740 & 8,793 & 27,704 \\
\hline NAUS & 1,900 & 1,650 & 13,136 & 35,582 \\
\hline POAL & 1.561 & 1,400 & 10,321 & 27.877 \\
\hline POVE & 1.788 & 1,600 & 10.559 & 31,174 \\
\hline PPTE & 1,894 & 1,742 & 8,024 & 32,833 \\
\hline PRMA & 2,035 & 1.834 & 9.844 & 27,123 \\
\hline RECF & 2,031 & 1,897 & 6.640 & 32,515 \\
\hline UFPR & 1,715 & 1,566 & 8,669 & 27,338 \\
\hline
\end{tabular}

Pode-se observar na Tabela 2 que a melhoria máxima ao longo do ano de 2013 variou de aproximadamente 23\% (BRAZ) a 37\% (BELE). A melhoria média para as estações do ano ao aplicar as correções do LPIM em relação ao GIM variaram de aproximadamente $7 \%$ (RECF) à $13 \%$ (NAUS). É importante destacar que dentre as estações que fazem parte da estimativa do VTEC no GIM, ou seja, estações BRAZ, POVE, RECF e UFPR, somente a estação BRAZ apresentou baixo nível médio anual de melhoria, atingindo aproximadamente 4\%, porém com máxima melhoria anual de aproximadamente $23 \%$.

No caso do processamento com a mesma estratégia de peso ao utilizar o GIM e LPIM, a estimativa de precisão das coordenadas obtidas a partir da Matriz de Variância e Covariância (MVC) dos parâmetros apresentam valores idênticos. Contudo, neste caso é necessário considerar que a MVC dos parâmetros é multiplicada pelo fator de variância a priori adotado, desde que o ajustamento seja aceito no teste estatístico Qui-quadrado, pois assim, pode-se considerar o fator de variância a priori estatisticamente igual ao fator de variância a posteriori.

Com base nestas informações e no nível de melhoria no posicionamento proporcionado pelo uso do LPIM em relação ao GIM, os dados de quatro estações (NAUS, POAL, PPTE e RECF) foram processados considerando na modelagem estocástica a incerteza dos mapas da ionosfera (ver seção 2), o que é apresentado na próxima seção. Na Figura 6 os valores do EMQ 3D são mensurados no eixo y à esquerda, cujos valores estão em metros, e o eixo y da direita apresenta os valores de melhoria percentual. 


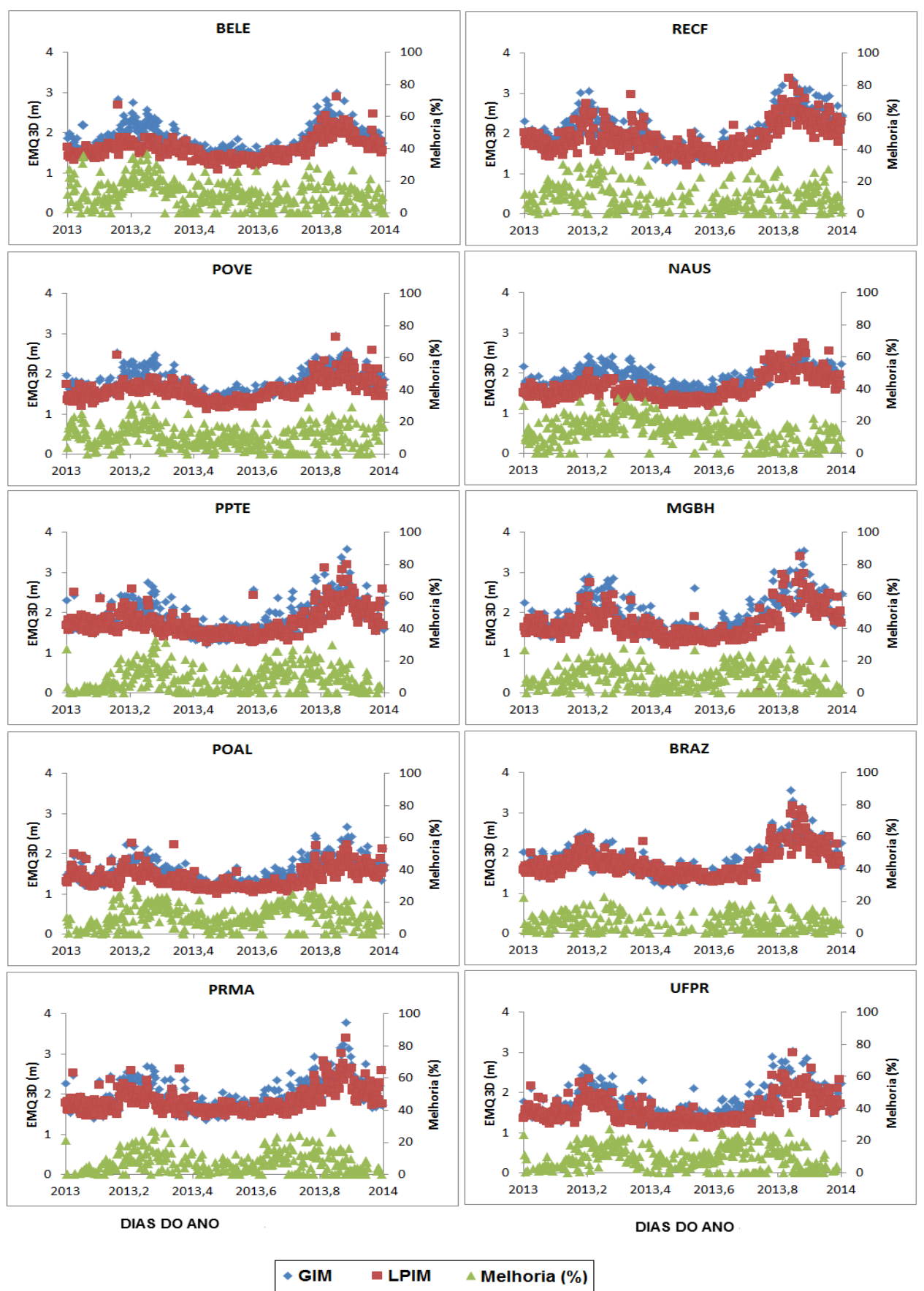

Figura 6: Série temporal do EMQ 3D e do melhoramento aplicando os mapas do GIM e LPIM sem ponderação.

Na Figura 6 verifica-se que, em geral, a aplicação do LPIM forneceu menores valores de EMQ $3 \mathrm{D}$ durante a maioria dos dias do ano de 2013. Nota-se o efeito sazonal na série temporal do EMQ 3D relacionado com as épocas do ano, onde os valores máximos podem ser observados nos meses de Fevereiro a Abril e de Setembro a Outubro, que correspondem as estações do ano verão e primavera, respectivamente, enquanto que os valores minimos podem ser observados entre os meses de Maio a Agosto, referente às estações de outono e inverno. Para os casos de máxima atividade ao longo do ano, os níveis de melhoria do LPIM em relação ao GIM são maiores atingindo a ordem de até $20 \%$, para algumas estações. 


\subsection{Aplicação do GIM e LPIM no PP considerando a incerteza do mapa ionosférico}

Os dados GPS de pseudodistâncias das estações NAUS, POAL, PPTE e RECF para o ano de 2013 foram processados no modo PP considerando a precisão das observações conforme apresentado na equação 7 (seção 2). Nesse caso, a precisão do código C/A foi adotada com valor fixo de 0,5 m e adicionada pelos valores de 0,7 TECU $(\sim 0,112 \mathrm{~m})$ e 0,6 TECU $(\sim 0,096 \mathrm{~m})$, respectivamente para a utilização do GIM e LPIM. Estes valores foram escolhidos de forma empírica após experimentos e considerou-se a precisão do LPIM em torno de $10 \%$ melhor que a precisão do GIM tendo em vista o nível médio anual de melhoramento apresentado na seção 3.1.

A Tabela 3 apresenta a média do EMQ 3D considerando todos os dias do ano 2013 (EMQ 3D anual), bem como o melhoramento médio e máximo ao longo do ano para o caso do processamento com diferentes estratégias de ponderação.

Tabela 3: EMQ 3D anual e o melhoramento no PPP aplicando os mapas do GIM e LPIM

\begin{tabular}{|c|c|c|c|c|}
\hline \multirow{2}{*}{ ESTAÇÄO } & \multicolumn{2}{|c|}{ EMQ 3D MÉDIO (m) } & \multirow{2}{*}{$\begin{array}{l}\text { MELHORIA } \\
\text { MÉDIA (\%) }\end{array}$} & \multirow{2}{*}{$\begin{array}{l}\text { MELHORIA } \\
\text { MAXIMA ( } \%\end{array}$} \\
\hline & GIM & LPIM & & \\
\hline NAUS & 1.894 & 1.639 & 13.461 & 35,603 \\
\hline POAL & 1.563 & 1.400 & 10.453 & 28,032 \\
\hline PPTE & 1,889 & 1,738 & 8,014 & 33,004 \\
\hline RECF & 2,029 & 1.886 & 7.053 & 32,922 \\
\hline
\end{tabular}

Ao comparar os valores do EMQ 3D médio obtido no processamento com ponderação (Tabela 3) e sem ponderação (Tabela 2), verifica-se que valores foram semelhantes apresenta-se valores pouco menores para o caso de ponderação. O nível de melhoria médio e máximo do LPIM em relação ao GIM no caso com ponderação também é bastante semelhante ao caso sem ponderação. A análise da precisão das coordenadas estimadas em cada época foi realizada para a estação NAUS considerando os dias GPS 58 (ionosfera ativa) e 159 (ionosfera calma) de 2013.

A MVC de parâmetros foi multiplicada pelo fator de variância a posteriori em cada época assumindo que o ajustamento foi aceito no teste estatístico Qui-quadrado. A Figura 7 apresenta a série temporal diária das precisões das coordenadas, as quais foram propagadas para o SGL. 


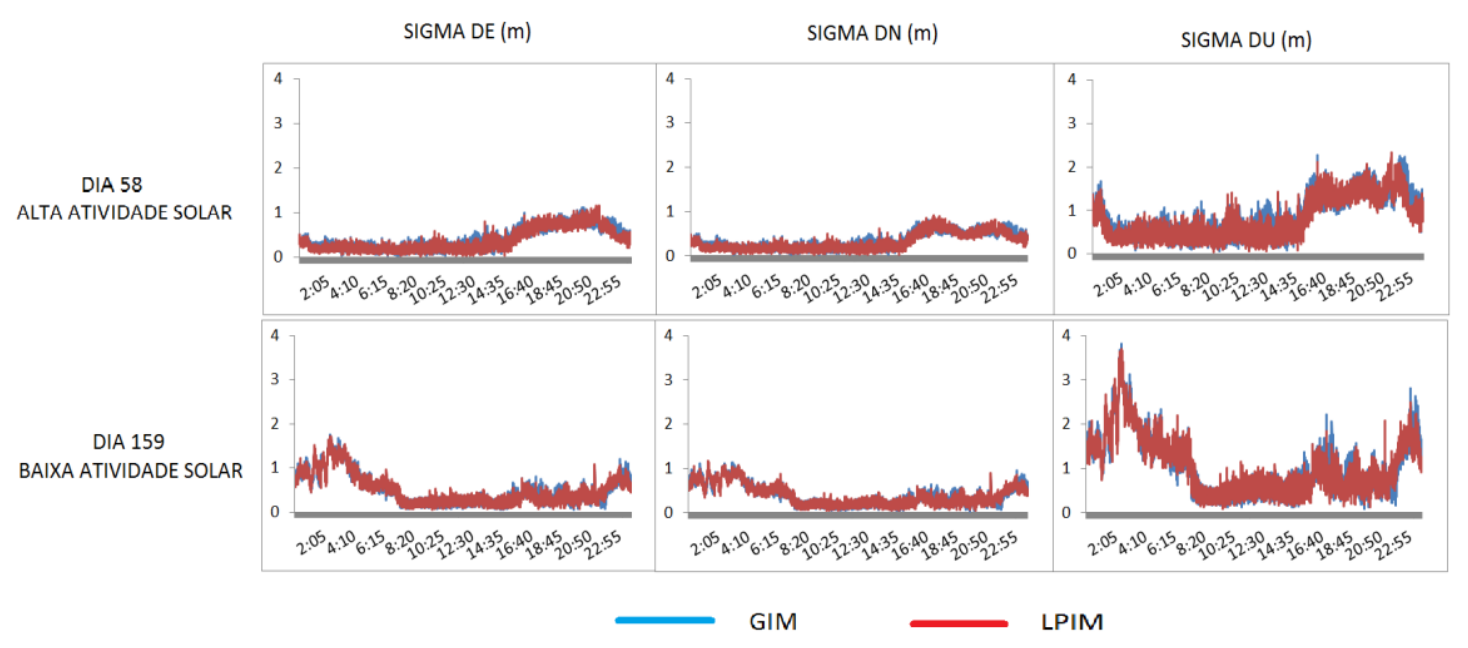

Figura 7: Serie temporal diária da precisão em DE, DN e DU para a estação NAUS no posicionamento com diferente estratégia de ponderação para os dias GPS 58 e 159.

A Tabela 4 apresenta a média diária das precisões no SGL ao aplicar o GIM e LPIM no posicionamento com diferentes estratégias de ponderação, bem como as melhorias percentuais.

Tabela 4: Precisões nas componentes DE, DN e DU (m) e o melhoramento (\%) no PPP aplicando os mapas do GIM e LPIM

\begin{tabular}{c|c|c|c|c}
\hline \multirow{2}{*}{$\begin{array}{c}\text { ESTAÇÄO - NAUS } \\
\text { DIA 58 }\end{array}$} & MAPAS & $\sigma \mathrm{DE}(\mathrm{m})$ & $\sigma \mathrm{DN}(\mathrm{m})$ & $\sigma \mathrm{DU}(\mathrm{m})$ \\
\hline \multirow{2}{*}{\begin{tabular}{c} 
ALTA ATIVIDADE SOLAR \\
\cline { 2 - 5 }
\end{tabular}} & GIM & 0,507 & 0,398 & 1,065 \\
\cline { 2 - 5 } & MELHORIA (\%) & 1,414 & 1,425 & 1,647 \\
\hline \multirow{2}{*}{$\begin{array}{c}\text { DIA 159 } \\
\text { BAIXA ATIVIDADE SOLAR }\end{array}$} & GIM & 0,412 & 0,338 & 0,869 \\
\cline { 2 - 5 } & LPIM & 0,386 & 0,315 & 0,808 \\
\cline { 2 - 5 } & MELHORIA (\%) & 6,477 & 6,797 & 6,989 \\
\hline
\end{tabular}

A partir dos resultados apresentados verifica-se que ao aplicar à correção ionosférica advinda do LPIM as melhorias nas precisões das coordenadas para o dia com ionosfera ativa (dia 58) foram de aproximadamente $1,41 \%, 1,42 \%$ e $1,66 \%$, respectivamente para as componentes DE, DN e DU. No caso do dia com ionosfera mais calma (dia 159), os níveis de melhoria foram maiores atingindo valores de aproximadamente $6,48 \%, 6,8 \%$ e 6,99\%, respectivamente para as componentes DE, DN e DU. A estratégia de ponderação aplicada especificamente para a utilização de cada mapa no processamento dos dados GPS é importante para o usuário que não disponha de coordenadas conhecidas da estação para avaliação, pois assim, será possível melhor avaliar a incerteza estimada das coordenadas. É importante ressaltar que este é o caso geral em que tais modelos serão utilizados. 


\section{Conclusões}

O principal objetivo deste trabalho foi avaliar a acurácia do posicionamento absoluto com a aplicação das correções ionosféricas advindas de mapas globais (GIM) e regionais (LPIM).

Foram realizados processamentos no modo absoluto utilizando dados GPS de dez estações da RBMC distribuídas ao longo do Brasil. A análise diária considerando dados GPS da estação NAUS para os dias 76 (ionosfera ativa) e 78 (ionosfera calma) de 2013 mostrou que a aplicação do LPIM proporcionou melhorias de aproximadamente $29 \%$ e $17 \%$ (ver Tabela 1) na componente altimétrica (DU), respectivamente, para os dias considerados. Para o EMQ 3D diário as melhorias com a utilização do LPIM em relação o GIM foram de aproximadamente $29 \%$ e $18 \%$, respectivamente, para os dias 76 e 78 . No caso do processamento para as dez estações no ano de 2013, a aplicação da correção ionosférica advinda do LPIM proporcionou melhoria máxima anual em termos de EMQ 3D variando de aproximadamente $22 \%$ (estação BRAZ) até 37\% (estação BELE) (ver Tabela 2).

Ao considerar a incerteza do VTEC do GIM ou do LPIM na modelagem estocástica do posicionamento, a precisão adotada para o VTEC após diversos experimentos foi, respectivamente, de 0,6 e 0,7 TECU, o que representa algo em torno de $10 \%$ melhor no caso da precisão do LPIM em relação ao GIM. Neste caso, a estimativa da precisão das coordenadas foi analisada para os dias 58 e 159 de 2013 na estação NAUS e as melhorias no uso do LPIM em relação ao GIM foram, respectivamente, de aproximadamente $2 \%$ e $7 \%$ na precisão da componente altimétrica $(\sigma D U)$ (ver Tabela 4$)$.

Com base nos experimentos realizados neste trabalho conclui-se que a utilização dos mapas regionais proporciona melhorias significativas em relação ao uso de mapas globais no posicionamento absoluto com máxima anual variando de $22 \%$ a $37 \%$. O nível de melhoria é influenciado pela localização geográfica da estação e pelo fato da utilização de dados que façam parte ou não da estimativa do VTEC nos modelos ionosféricos (GIM e LPIM) utilizados. A introdução de incertezas dos mapas regionais e globais proporciona ao usuário GPS valores mais realísticos da qualidade dos parâmetros, o que é fundamental para as mais diversas aplicações, uma vez que os usuários não dispõem de coordenadas conhecidas da estação para realizar suas análises.

\section{AGRADECIMENTOS}

Os autores agradecem ao CNPQ pelo apoio financeiro em forma de projeto Universal (Processo: 475775/2013-9) e pelo apoio em forma de bolsa de mestrado junto ao Programa de PósGraduação em Ciências Geodésicas e Tecnologias de Geoinformação da UFPE.

\section{REFERÊNCIAS BIBLIOGRÁFICAS}

Aguiar, C. R. Modelo Regional da Ionosfera (Mod_Ion): Implementação em Tempo Real. 2005. 159 f. Dissertação (Mestrado em Ciências Cartográficas) - Faculdade de Ciências e Tecnologia, Universidade Estadual Paulista, Presidente Prudente. 2005. 
Boehm, J.; Niell, A.; Tregoning, P.; Schuh, H. Global Mapping Function (GMF): A new empirical mapping function based on numerical weather model data. Geophys. Res. Lett. 33: L07304. doi: 10.1029/2005GL025546, 2006.

Brunini, C. A. Global ionospheric model from GPS measurement, Ph.D. thesis, Facultad de Ciencias Astronómicas y Geofísicas, Universidad Nacional de La Plata, La Plata, Argentina, 1998.

Brunini, C.A.; Meza, A.; Gende, M.; Azpilicueta, F. South American regional ionospheric maps computed by GESA: a pilot service in the framework of SIRGAS, Advances in Space Research, doi: 0.1016/j.asr.2007.08.041. 2008

Camargo, P. O. Modelo Regional da Ionosfera para uso em posicionamento com receptores de uma frequência. 191 p. Tese (Doutorado em Ciências Geodésicas) - Setor de Ciências da Terra, Universidade Federal do Paraná, Curitiba. 1999.

CODE (2014). Center for Orbit Determination in Europe, Astronomy Institute University of Berne (AIUB). http://aiuws.unibe.ch/ionosphere/. Acesso em: Dezembro de 2014.

CPLAT (Centro de Processamento de La Plata) - SIRGAS. 2014. http://cplat.fcaglp.unlp.edu.ar/ . Acesso: Novembro de 2014.

Dach, R. et al. (Ed.). Bernese GPS software version 5.0: user manual. Astronomical Institute, University of Bern, 2007.

Hofmann-Wellenhof, B.; Lichtenegger, H.; Collins, J. Global Positioning System: theory and practice. 5.ed. New York: Springer-Verlag, 2001. 382p.

IGS - International GNSS Service. Workshop IGS 2014. Disponível em: 〈http://www.rtigs.net〉. Acesso em: Agosto de 2014.

Klobuchar, J. A. C. Ionospheric effects on GPS. In PARKINSON, B. W.; SPILKER JR., J. J. Global Positioning System: Theory and applications. Washington: American Institute of Aeronautics and Astronautics, 1996. v. 2, Cap. 12.

Leandro, R. F. Precise Point Positioning With GPS: A New Approach for Positioning, Atmospheric Studies and Signal Analysis. 2009. PhD Thesis - University of New Brunswick, Canadá. 2009.

Leick, A. GPS satellite surveying. 435 p. $3^{\mathrm{a}}$ ed.: New York: John Wiley \& Sons, 2004.

Liu, G. C. Ionosphere weighted global positioning system carrier phase ambiguity resolution. 2001. 157 f. M.Sc. dissertation. Department of Geomatics Engineering - The University of Calgary, Calgary, Alberta, Canada. 2001.

Marques, H. A. PPP em tempo real com estimativa das correções dos relógios dos satélites no contexto de rede GNSS. 245 f. Tese (Doutorado em Ciências Cartográficas) - Faculdade de Ciências e Tecnologia, Universidade Estadual Paulista, Presidente Prudente. 2012.

Marques, H. A.; Monico, J. F. G.; Marques, H. A. S.; Aquino, M. Análise dos Efeitos Ionosféricos de Ordem Superior no Ciclo Solar 24 e Influência no Posicionamento GNSS Absoluto. Boletim de Ciências Geodésicas, v. 20, nº 4, p.879-901, 2014. 
Matsuoka, M. T. Avaliação de funções para modelagem do efeito da refração ionosférica na propagação dos sinais GPS. 164 p. Dissertação (Mestrado em Ciências Cartográficas) Programa de Pós-Graduação em Ciências Cartográficas, Faculdade de Ciências e Tecnologia, Universidade Estadual Paulista, Presidente Prudente. 2003.

Matsuoka, M. T. Influência de diferentes condições da ionosfera no posicionamento por ponto com GPS: avaliação na região brasileira. 264 p. Tese (Doutorado em Ciências Cartográficas) Programa de Pós-Graduação em Ciências Cartográficas, Faculdade de Ciências e Tecnologia, Universidade Estadual Paulista, Presidente Prudente. 2007.

Monico, J. F. G. Posicionamento pelo GNSS: Descrição, Fundamentos e Aplicações. 476 p. $2^{\text {a }}$ ed. São Paulo: UNESP, 2008.

Sapucci, L. F.; Machado, L. A. T.; Monico, J. F. G. Previsões do Atraso Zenital Troposférico para a América do Sul: Variabilidade Sazonal e Avaliação da Qualidade. Revista Brasileira de Cartografia. N.58/3, 2006.

Schaer, S. Mapping and predicting the earth's ionosphere using the Global Positioning System. 1999. 205p. Ph.D. dissertation. University of Bern, Bern, Switzerland.

Schaer, S.; Gurtner, W. IONEX: The IONosphere Map Exchange Format Version 1. ESA/ESOC, Darmstadt, Germany, 1998.

Seeber, G. Satellite geodesy: foundations, methods, and applications. 2. ed. Berlin, New York: Walter de Gruyter. 589p. 2003.

Silva, H. A. Avaliação de modelos estocásticos no posicionamento GNSS. 2009. $108 \mathrm{f}$. Dissertação (Mestrado em Ciências Cartográficas) - Faculdade de Ciências e Tecnologia, Universidade Estadual Paulista, Presidente Prudente. 2009.

SIRGAS - SISTEMA DE REFERÊNCIA GEOCÊNTRICO PARA AS AMÉRICAS. 2014. Disponível em: http://www.sirgas.org. Acesso: nov/2014.

Van Bree, R. J.P.; Tiberius, C. C. J. M. Real-time single-frequency precise point Positioning: Accuracy assessment. GPS Solution. $\mathrm{n}^{\mathrm{o}}$ 16, p.259-266. 2012.

Wilson, B.; Yinger, C.; Feess, W.; Shank, C.; New and Improved: The Broadcast Interfrequency Biases, In: GPS World, USA, v.10 n.9, p. 56-66, set., 1999.

Recebido em janeiro de 2015. Aceito em março de 2015. 